\title{
Generating Helices in Nature
}

\section{Citation}

Forterre, Yoel, and Jacques Dumais. 2011. Generating Helices in Nature. Science 333, no. 6050: $1715-1716$.

\section{Published Version}

doi:10.1126/science.1210734

\section{Permanent link}

http://nrs.harvard.edu/urn-3:HUL.InstRepos:12724042

\section{Terms of Use}

This article was downloaded from Harvard University's DASH repository, and is made available under the terms and conditions applicable to Other Posted Material, as set forth at http:// nrs.harvard.edu/urn-3:HUL.InstRepos:dash.current.terms-of-use\#LAA

\section{Share Your Story}

The Harvard community has made this article openly available.

Please share how this access benefits you. Submit a story.

Accessibility 


\title{
Generating Helices in Nature
}

\author{
Yoel Forterre $^{1}$ and Jacques Dumais ${ }^{2}$ \\ ${ }^{1}$ Institut Universitaire des Systèmes Thermiques et Industriels, Centre National de la \\ Recherche Scientifique, Aix-Marseille Université, 13453 Marseille cedex, France. \\ email: yoel.forterre@polytech.univ-mrs.fr \\ ${ }^{2}$ Department of Organismic and Evolutionary Biology, Harvard University, \\ Cambridge, MA 02138. \\ email: jdumais@oeb.harvard.edu
}

Helical structures arise in many different contexts and scales in nature, from biological macromolecules (DNA, bacterial flagella, viral capsids) to macroscopic organs such as seashells, horns, plant tendrils and seed pods (see the figure). In material science, the familiar screws and helical springs can now be synthesized at the microscale using selforganized nanoparticles or thin films, offering new opportunities to generate materials with unique chiral properties $(i, i i)$. From a geometrical point of view, helical shapes may be classified in two main families: $i$ ) helicoids, i.e. twisted helices with saddle-like curvature and straight centerline, and ii) cylindrical helices with cylindrical curvature and helical centerline. The mechanisms underlying the formation of helicoid or helical ribbons and the nature of the transition between these two limiting cases have been the focus of some recent studies (iii,iv,v,vi). Yet, one open question is the extent to which chirality in these structures reflects the chirality of their molecular constituents or emerges from global geometrical constraints. On page $\mathrm{xx}$ of this issue, Armon et al. explore the rich phenomenology associated with slender strips made of mutually opposing "molecular" layers; taking a singular botanical structure, the Bauhinia seed pod, as their inspiration. Using experiments on a physical system mimicking the pod structure and a mathematical model based on the mechanics of thin sheets, they show that a single ingredient, namely a flat strip with a saddle-like intrinsic curvature, is sufficient to generate a wide variety of helical shapes, from twisted helices to cylindrical ribbons, including many intermediate shapes.

To illustrate Armon et al.'s findings, we use the same paper models employed by botanists to explain, more than 100 years ago, the helical shape of the Bauhinia pod and related plant structures (vii,viii). The wood fibers within a piece a paper lie nearly parallel to each other providing the sheet with anisotropic material properties. When dipped in water, the fibers swell preferentially in their transverse direction. By putting two such sheets back-to-back but with their fiber directions at right angle to each other (see figure), it is possible to mimic the structure of many plant tissues such as the seed pods studied by Armon and coworkers (7). The antagonistic response of the double layer leads to a rich phenomenology of behaviors. For a wide strip cut along one of the principal fiber directions (i.e. $0^{\circ}$ or $90^{\circ}$ ), one observes pure bending either along or transverse to the long axis of the strip. For a strip cut inclined with respect to the fiber direction, one observes chiral shapes either in the form of helicoids or helical ribbons. 
The central feature of the bilayer paper models is the opposite intrinsic curvatures: when the strip swells, it 'wants' to bend in two opposite and perpendicular directions. For an initially flat strip, this saddle-like configuration cannot be achieved without stretching the sheet, meaning that elastic energy is stored in the system. To find the equilibrium shape of such 'mechanically incompatible' strips, Armon et al. developed a general and elegant mathematical framework where the configuration of the strip is fully characterized by two 2D tensors: a curvature tensor, describing the local curvature of the sheet and a metric tensor, describing the distance between points on the surface of the strip. They then minimize the total elastic energy of the strip written as the sum of bending and stretching energy. Armon et al's main result is that all the variety of helical shape shown in the figure are controlled by only two dimensionless parameters: the direction $\theta$ between the strip's long axis and the direction of intrinsic curvature, and the dimensionless width $\tilde{w}=w /\left(t / \kappa_{0}\right)^{1 / 2}$, where $w$ is the width of the strip, $t$ its thickness and $\kappa_{0}$ its intrinsic curvature. For $\theta=45^{\circ}$, 'narrow' strips (small width, large thickness, small intrinsic curvature) form helicoids whereas 'large' strips (large width, small thickness, large intrinsic curvature) form helical ribbons. Experiments performed on elastic bilayers composed of stretched latex sheets glued together quantitatively agree with the model predictions for a wide range parameters. In particular, the pitch and radius of the helices are predicted, as well as the smooth transition between helicoids and helical ribbons. Interestingly, in the 'wide' regime, two states having the same elastic energy coexist for a given value of $(\theta, \tilde{w})$ (see figure). To pass from one state to another requires crossing an energy barrier, which involves the slow storing and rapid release of elastic energy. This snap-through mechanism in bodies with opposite intrinsic curvatures is found in some plants to speed up their movement $(i x)$ and is used in deployable structures such as tape springs $(x)$.

Armon et al.'s study accompanies several works published in the last year on helical shape formation in different fields and contexts that, together, underlie the robustness and universality of the simple mechanism proposed by Armon et al. For example, Sawa et al. (xi) performed experiments on thin strip made of twist-nematic elastomer (soft materials possessing both the elastic properties of rubber and the orientational properties of liquid crystals) and showed a similar transition between helicoid and helical ribbons as the system width increases. To model their system, they introduced a term in the elastic energy accounting for a spontaneous twist at the molecular scale, an approach frequently used to describe chiral biological macromolecules and membranes. Chen et al. (xii) developed a framework to predict the helical shape of narrow ribbons with arbitrary intrinsic curvature, taking surface stress anisotropy as the main ingredient of anisotropy. Formally, the generic approach of Armon et al. should be applicable to all these different situations, as it does not rely on the specific knowledge of the microscopic interaction. The Bauhinia seeds pod and their mechanical analogues clearly show how chiral shapes can emerge from global geometrical constraints without any chirality at the microscopic level. In the paper bilayers, the handedness of the helix is determined by the orientation of the principal fiber directions with the long axis of the strip, and can be reversed by simply cutting the strip at an angle below or above $90^{\circ}$. That this versatility can be achieved with a mere piece of paper should convince anyone of the power of this approach to build materials with exciting new properties. 


\section{References}

i. Y. Qiao et al., Nanoletters 9, 4500 (2009).

ii. Y. Lin et al., Chem. Mat. 22, 6711 (2010).

iii. R. Oda et al., Nature 399, 566 (1999).

iv. R.L.B. Selinger et al., Phys. Rev. Let. 93, 158103 (2004).

v. R. Ghafouri, R. Bruinsma, Phys. Rev. Let. 94, 138101 (2005).

vi. N. Chouaieb et al., Proc. Nat. Acad. Sci. USA 103, 9398 (2006).

vii. C. Steinbrinck, Biologisches Centralblatt 26, 657 (1906).

viii. J. Verschaffelt, Maandlab voor Natuurwet. 17, 1 (1891).

ix. Y. Forterre et al., Nature 433, 421 (2005).

x. K. Seffen et al., Int. J. Mech. Sci. 42, 2055 (2000).

xi. Y. Sawa et al., Proc. Nat. Acad. Sci. USA 108, 6364 (2011).

xii. Z. Chen et al., Appl. Phys. Let. 98, 011906 (2011).

The coiling of pods and paper models. (A) An initially straight seed pod was split to show the strong curvature stored in the two valves. (B) Protocol used to prepared paper models reproducing the coiling behavior of plant structures. (C) The geometry of paper models for various values of the two control parameters: the dimensionless width and the fiber angle. Note the reversal of the blue and yellow faces for fiber angles exceeding $45^{\circ}$ and the transition from helicoid to cylindrical ribbons as the width is increased. 